\title{
Bose condensation in a model microcavity
}

\author{
P. R. Eastham and P. B. Littlewood \\ Theory of Condensed Matter, Cavendish Laboratory, Cambridge, CB3 0HE. United Kingdom.
}

(July 19, 2021)

\begin{abstract}
We study the equilibrium properties of a system of dipoleactive excitons coupled to a single photon mode at fixed total excitation. Treating the presence or absence of a trapped exciton as a two-level system produces a model that is exactly soluble. It gives a simple description of the physics of polariton condensation in optical cavities beyond the low-density bosonic regime.

71.35.Lk, 71.36.+c, 71.10.Li, 73.20.Dx
\end{abstract}

The coupled exciton-photon modes of a microcavity were first observed by Weisbuch et al. [1]. Since at low densities excitons are bosons, at low densities coupled exciton-photon modes are also bosons. These bosonic excitations are known as cavity polaritons.

Considerable effort has been devoted to seeking experimental evidence for the bosonic nature of cavity polaritons. This evidence has been sought in the nonequilibrium behavior of microcavities [2 8]. In this paper we will consider the simpler equilibrium problem, in particular the possibility of a Bose condensate of cavity polaritons.

Polaritons are not conserved particles, so there is ultimately no equilibrium condensate. We may, however, treat polaritons as conserved particles if their lifetime is much longer than the time required to achieve thermal equilibrium at a fixed polariton number. We will study such a quasi-equilibrium limit in a model microcavity.

While the theory of weakly-interacting bosons is well understood, it is far from obvious that this theory is appropriate to the cavity polariton condensate. The concept of a polariton [9] is only valid in linear response; it is not valid for a substantial occupation of the excitons. Finite exciton densities introduce saturation of the electronic states, so the excitons cannot be treated as bosons. In general, finite densities of excitons also lead to exciton-exciton interactions, which can produce dephasing and ionization of the excitons [10]. By considering a situation in which neither dephasing nor ionization are relevant, we will show how to generalize the concept of a polariton to include saturation of the exciton states. Saturation alone does not preclude Bose condensation.

We assume that the relevant electronic excitations in the microcavity are localized, physically separated, single excitons. Thus we can neglect the Coulomb interaction between excitons localized on different sites. We further assume that, because of the tiny effective mass of a cavity $\operatorname{photon}\left(\sim 10^{-5} m_{e}\right.$ for a $1000 \AA$ cavity $)$, there is only a single relevant photon mode in the cavity.

These assumptions lead us to consider the well-known Dicke model [11]. This consists of a single mode of the photon field, dipole coupled to a set of $N$ localized twolevel oscillators. Each two-level oscillator represents one exciton state, localized on site $n$ with an energy $E_{g}(n)$. These exciton states are composed of conduction and valence electrons with fermionic annihilation operators $b_{n}$ and $a_{n}$ respectively; these fermions are subject to the local constraints $b_{n}^{\dagger} b_{n}+a_{n}^{\dagger} a_{n}=1$. For brevity, we suppress the site index $n$ on the fermion operators. Making the rotating wave approximation, we consider the Hamiltonian

$$
\begin{aligned}
H= & \sum \frac{E_{g}(n)}{2}\left(b^{\dagger} b-a^{\dagger} a\right)+\omega_{c} \psi^{\dagger} \psi \\
& +\frac{g}{\sqrt{N}} \sum\left(b^{\dagger} a \psi+\psi^{\dagger} a^{\dagger} b\right) .
\end{aligned}
$$

$\psi$ is the bosonic annihilation operator for the cavity mode and the summations are over the site index $n$.

The operator $\sigma_{z}=\frac{1}{2} \sum\left(b^{\dagger} b-a^{\dagger} a\right)$ measures the electronic excitation; such excitations are created by the operator $\frac{1}{\sqrt{N}} \sum b^{\dagger} a$. They are approximately bosonic provided we remain near to the bare ground state, so that $\left\langle\sigma_{z}\right\rangle \approx-N / 2$. In this limit equation (1]) becomes two coupled harmonic oscillators and we recover the usual bosonic polaritons.

Away from $\left\langle\sigma_{z}\right\rangle=-N / 2$ saturation of the electronic states becomes relevant and the excitations are no longer bosonic. However, we can still define a polariton: it is the quantum of excitation of the non-linear coupled system. With this definition the polariton number operator is $L=\psi^{\dagger} \psi+\sigma_{z}$, which is a conserved quantity for the Hamiltonian (1).

The thermal equilibrium of the Dicke model was originally solved by Hepp and Lieb 12]. Here we are interested in the quasi-equilibrium problem posed by (1) at constant total excitation $L$, a generalization obtained by adding a chemical potential to constrain $L$. Thus at $T=0$ the relevant free energy is $\left\langle H-\mu_{\mathrm{ex}} L\right\rangle$, where $\mu_{\mathrm{ex}}$ is the chemical potential for excitations.

To keep the number of parameters to a minimum we restrict ourselves to the uniform case $E_{g}(n)=E_{g}$. There are then only three parameters in the problem: the temperature $\left(k_{B} \beta\right)^{-1}$, the dimensionless detuning $\Delta=\left(\omega_{c}-E_{g}\right) / g$, and the excitation density $\rho_{\mathrm{ex}}=\langle L\rangle / N$. Generalizing our solution to include a distribution of exciton energies is straightforward. 


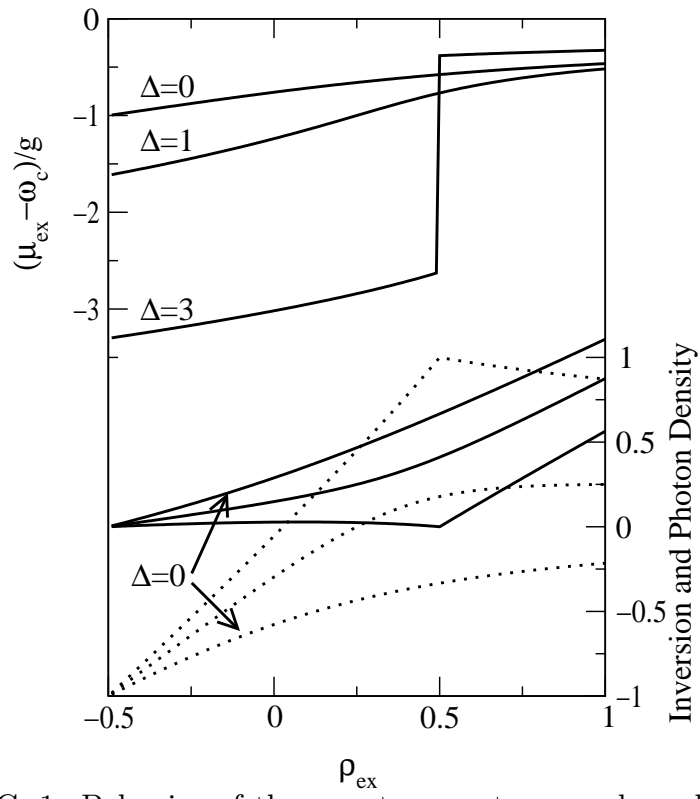

FIG. 1. Behavior of the zero-temperature condensed solution as a function of excitation density. Upper section (left axis): chemical potential, for detunings $\Delta=0,1$ and 3 . Lower section (right axis): photon density(solid lines) and inversion density $2\left\langle\sigma_{z}\right\rangle / N$ (dotted lines), for the same three detunings. The inversion increases with increasing $\Delta$, while the photon density decreases.

Following Kiry'anov and Yarunin's work [13] on the unconstrained problem, we solve the model using the large- $N$ expansion of a coherent-state path-integral. This expansion is possible because all the exciton states couple to a single mode of the photon field. More detailed derivations, as well as a variational approach based on the work of Comte and Nozières [14] on the exciton condensate, will be presented in a future publication.

By integrating out the fermions and rescaling the photon field, the grand partition function for (1i) can be represented as an imaginary-time path-integral over the photon field of the form $Q \propto \int \mathcal{D} \psi \exp \left(-N S_{\text {eff }}\right)$. In the thermodynamic limit $N \rightarrow \infty$ the partition function is dominated by those paths $\psi_{0}(\tau)$ which minimize the action $S_{\text {eff. }}$. The Euler-Lagrange equation for $S_{\text {eff }}$ is a self-consistency condition which relates $\psi_{0}(\tau)$ to the equilibrium polarization of a two-level system in the selfconsistent field $\psi_{0}(\tau)$. It takes the form

$$
\left(\partial_{\tau}+\tilde{\omega}_{c}\right) \psi_{0}(\tau)+g\langle\bar{a}(\tau) b(\tau)\rangle=0,
$$

with $\tilde{\omega}_{c}=\omega_{c}-\mu_{\mathrm{ex}}$.

The order parameter of the polariton condensate is the polarization of either the photon field or the exciton field. Although there are two physically distinct order parameters, we see from equation (2) that they are coupled by the dipole interaction. It is the dipole interaction which favors the formation of a condensate.

We assume that the extremal trajectories are independent of $\tau, \psi_{0}(\tau)=\psi_{0}$. We can then use the familiar eigenstates 15] of a two-level system in a static external field to calculate the polarization term on the right of equation (2). A constrained thermal population of these renormalized eigenstates leads to a BCS-like equation for $\psi_{0}$,

$$
\tilde{\omega}_{c} \psi_{0}=\frac{g^{2} \psi_{0}}{2 E} \tanh (\beta E) .
$$

Here $E$ is the renormalized fermion energy $E=$ $\sqrt{\tilde{\varepsilon}^{2}+g^{2}\left|\psi_{0}\right|^{2}}$, with $\tilde{\varepsilon}=\left(E_{g}-\mu_{\text {ex }}\right) / 2$.

The chemical potential $\mu_{\mathrm{ex}}$ is related to the partition function $Q$ in the usual manner. For the condensed solutions the leading term in the expansion of $Q$ around the extremal trajectories gives

$$
\rho_{\mathrm{ex}}=\left|\psi_{0}\right|^{2}-\frac{1}{g^{2}} \tilde{\varepsilon} \tilde{\omega}_{c}
$$

while for the normal solution we have $\rho_{\mathrm{ex}}=$ $-[\tanh (\beta \tilde{\varepsilon})] / 2$.

At zero temperature (3) and (4) always have a condensed solution $\psi_{0} \neq 0$. This solution is illustrated in Fig. 1. The upper part of Fig. 1 1 shows the chemical potential as a function of excitation density for detunings $\Delta=0,1$ and 3 . In the low density limit, $\rho_{\mathrm{ex}}=-0.5$, we are describing a condensate of conventional bosonic polaritons, so the chemical potential is given by the usual polariton energy $\mu_{\mathrm{ex}}=\frac{1}{2}\left(\left(\omega_{c}+E_{g}\right)-g \sqrt{\Delta^{2}+4}\right)$. At high densities the electronic states are saturated and further excitation must be added as photons. Thus the chemical potential approaches the energy of the cavity mode. Between these two limits we find a discontinuity at $\rho_{\mathrm{ex}}=0.5$ if $\Delta>2$. The lower part of Fig. 1 illustrates the composition of the condensate, again for detunings $\Delta=0,1$ and 3 . Increasing detuning increases the electronic fraction of the condensate. In the high excitation limit the inversion approaches zero. This gives the maximum polarization of the electronic states and hence minimizes the dipole energy.

We study the stability of the solutions to (3) by considering the quadratic term $S_{2}$ in the functional Taylor series expansion of $S_{\text {eff }}$ around the extremal trajectory $\psi_{0}$. The kernel of $S_{2}, \mathcal{G}^{-1}$, is the inverse matrix Green's function for fluctuations of the photon field. We find $\mathcal{G}^{-1}$ by taking two functional derivatives of $S_{\text {eff }}$ and evaluating the result on the extremal trajectory $\psi_{0}$. This amounts to solving the Dyson-Beliaev equations [16] for the photon Green's functions. The self-energies are provided by the polarizability of a two-level system in the self-consistent field $\psi_{0}$.

The resulting expression for $\mathcal{G}^{-1}$ in the condensed phase is somewhat lengthy, so we do not reproduce it here. We find that the eigenvalues of $\mathcal{G}^{-1}$ in the condensed phase are, apart from the Goldstone mode, strictly positive provided $\tilde{\omega}_{c}>0$. This condition is automatically satisfied by the condensed solutions to (3). 


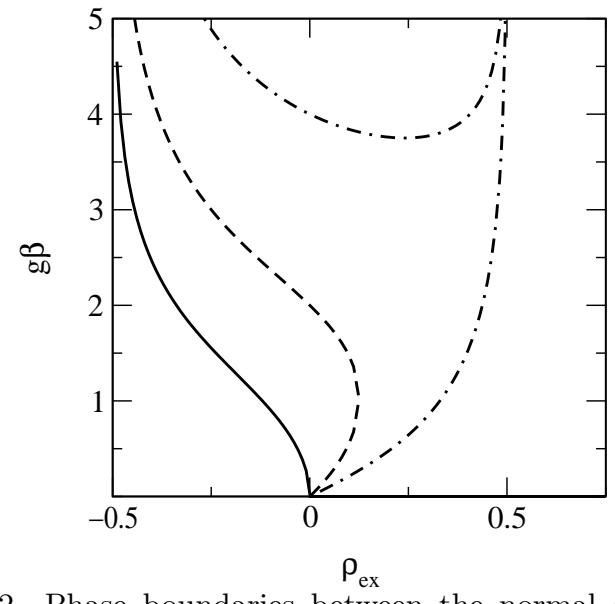

FIG. 2. Phase boundaries between the normal and condensed states for $\Delta=0$ (solid line), $\Delta=1$ (dashed line), and $\Delta=2$ (dot-dashed lines). The regions which include the lower left corner of the figure are normal.

To determine the phase diagram we assume a continuous transition between the normal and condensed states. The transition temperature $\left(k_{B} \beta_{c}\right)^{-1}$ is determined by requiring (3) and (4) to have a repeated root $\psi_{0}=0$. This gives two transition temperatures

$$
\beta_{c} g=\frac{4 \tanh ^{-1}\left(2 \rho_{\mathrm{ex}}\right)}{\Delta \pm \sqrt{\Delta^{2}-8 \rho_{\mathrm{ex}}}} .
$$

We also obtain (5) as the temperature corresponding to the onset of a low frequency instability of the normal state. Thus our assumption of a continuous transition is correct.

The phase boundary (5) is illustrated in Fig. 2 for detunings $\Delta=0,1$ and 2. For $\Delta \leq 0$ the phase diagram is straightforward. The transition temperature increases monotonically with density, reaching infinity at $\rho_{\mathrm{ex}}=0$. For $\Delta>0$ there is a region in which the condensate exists on both the high and low temperature sides of the normal state. When $\Delta \geq 2$ the condensate exists in two disconnected regions of the phase diagram.

This unusual phase diagram can be understood by considering the excitations of the normal state. The excitation energies follow in the usual manner from the locations of the poles of the Green's function. We write the action for fluctuations of the photon field about the normal state as $S_{2}=\beta \sum_{\omega_{n}} \delta \bar{\psi}\left(\omega_{n}\right) \mathcal{G}_{N}^{-1}\left(\omega_{n}\right) \delta \psi\left(\omega_{n}\right)$, where $\omega_{n}$ is a bosonic Matsubara frequency. The normal-state Green's function $\mathcal{G}_{N}$ takes the form

$$
\mathcal{G}_{\mathcal{N}}\left(\omega_{n}\right)=\frac{C_{+}}{i \omega_{n}+E_{+}}+\frac{C_{-}}{i \omega_{n}+E_{-}},
$$

with $E_{ \pm}=\left[\left(\omega_{c}+E_{g}\right) \pm g \sqrt{\Delta^{2}-8 \rho_{\mathrm{ex}}}\right] / 2-\mu_{\mathrm{ex}}$ and $C_{ \pm}= \pm\left(2 \tilde{\varepsilon}-E_{ \pm}\right) /\left(E_{-}-E_{+}\right)$. The excitation energies, measured from the chemical potential $\mu_{\mathrm{ex}}$, are $E_{ \pm}$.

The normal-state excitations are polaritons in the sense of Hopfield [9]: coupled modes involving the linear

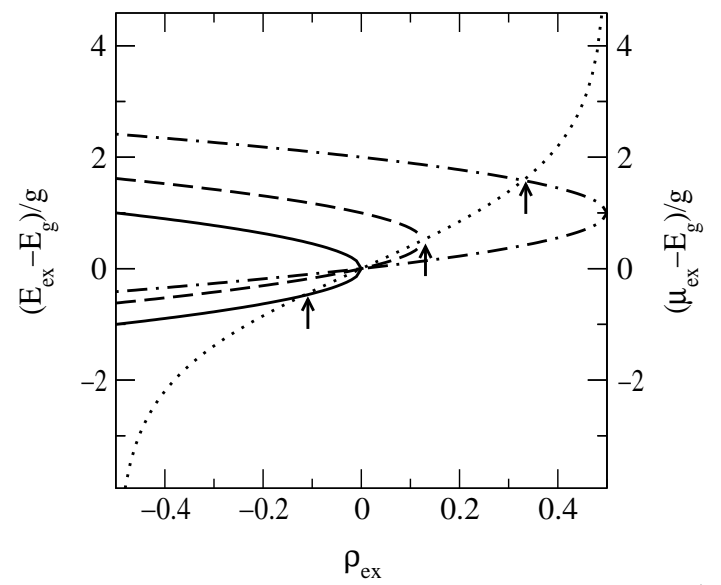

FIG. 3. Normal state excitation energies $E_{\text {ex }}$ for $\Delta=0$ ( solid curve), $\Delta=1$ (dashed curve), and $\Delta=2$ (dot-dashed curve), relative to the exciton energy $E_{g}$. The dotted curve is the normal state chemical potential for $\beta g=1$ on the same scale. The arrows mark the crossings which correspond to the onset of condensation for these three detunings at $\beta g=1$.

response of the electronic system around its equilibrium state. The gap in the spectrum is increased over the bare detuning $\Delta$ owing to the dipole coupling between the excitons and the cavity mode. The presence of excitation in the ground state, either driven by finite temperatures or by finite $\mu_{\mathrm{ex}}$, causes the two polariton branches to attract. This attraction can be understood in terms of an angular momentum representation [11] for the collective states of the electronic system. The excitation of the electronic states $\sigma_{z}$ forms the z-component of an angular momentum and their polarization forms a raising operator $\sigma_{+}$. Thus the polarizability of the electronic states is a maximum at $\left\langle\sigma_{z}\right\rangle=-N / 2$.

Figure 3 illustrates the excitation energies $E_{\text {ex }}=E_{ \pm}+$ $\mu_{\text {ex }}$ obtained from equation (6), for detunings $\Delta=0,1$ and 2 . On the same axis we plot the normal state chemical potential, given by the expression immediately below equation (1), for $\beta g=1$. Figure 3 should be compared with the $\beta g=1$ line of the phase diagram in Fig. 2 .

When $\Delta=0$ and $\rho_{\text {ex }}=-0.5$ the system is in the normal state. Increasing $\rho_{\text {ex }}$ populates the electronic excitations, increasing the chemical potential and decreasing the polariton splitting. Eventually the chemical potential crosses the lower polariton branch from below and the system condenses. In contrast, for $\Delta=1$ and 2 the chemical potential crosses the lower polariton branch at $\rho_{\text {ex }}=0$ without the condensate appearing. It is not until the chemical potential crosses the upper polariton branch that the transition occurs. This can be understood by considering the signs of the quasiparticle weights $C_{ \pm}$. A positive quasiparticle weight corresponds to absorption of an external field(particle-like excitations), whereas a negative quasiparticle weight corresponds to gain(holelike excitations). For $\rho_{\mathrm{ex}}>0$, the lower polariton branch has a negative weight: it has become hole-like, and must 
be below the chemical potential for stability. Note that for large $\Delta$ there is a particle-hole symmetry, so the region $-0.5<\rho_{e x}<0.5$ of the phase diagram is symmetric about $\rho_{\text {ex }}=0$.

In the condensed phase we find excitations with energies relative to the chemical potential $E_{ \pm}=$ $\pm \sqrt{\left(\tilde{\omega}_{c}+2 \tilde{\epsilon}\right)^{2}+g^{2}\left|\psi_{0}\right|^{2}}$, along with a Goldstone mode. The polariton condensate will produce incoherent luminescence or absorption at the energies $E_{ \pm}+\mu_{\mathrm{ex}}$ and coherent emission at $\mu_{\mathrm{ex}}$.

Since we expect polariton condensates to produce coherent light, a major issue experimentally will be distinguishing polariton condensates from semiconductor lasers. In semiconductor lasers excitons are conventionally assumed to be ionized. We list some aspects of the present work which should help to distinguish polariton condensation from lasing: (1)From Fig. 1, we see that the polariton condensate can exist without an inversion of the electronic system. (2)Approaching the condensation transition by increasing the excitation density we may observe a reduction of the normal-state excitation gap owing to the fermionic structure of the excitons. (3)The fermionic structure of the excitons shifts the coherent emission from the condensate away from the bosonic polariton energy. (4)The incoherent luminescence and absorption from the condensate exhibits a gap induced by the coherent photon field. In a conventional laser, this gap is destroyed by the very short relaxation time of the electronic polarization. Following [17], processes which destroy the electronic polarization could be incorporated into our solution. In superconductors, such processes produce a regime of gapless superconductivity 18, 19 where the order parameter survives without a gap in the excitation spectrum.

The applicability of our results to real microcavities is restricted by our neglect of exciton states with a significant wavefunction overlap. We have assumed that these states are at infinitely large energies. In reality, these states exist above an energy $E_{m}$. Thus our thermodynamic results are only valid when $E_{m}-\mu_{e x}$ is large compared with $\beta^{-1}$ and $g$. By considering Fig. 3, we deduce that realizing the phase diagram of Fig. 2 requires an energy gap $\Delta E=E_{m}-E_{0} \gg g$. This could occur in highly disordered materials with Frenkel-like excitons, such as organic semiconductors 20,21]. An energy gap $\Delta E$ could exist in inorganic quantum wells if the excitons move in a potential containing deep, well-separated traps, perhaps associated with interface islands 22 24. However, in both these cases it is likely that there will be several exciton states on each site, rather than the single state we have assumed.

If $\Delta E$ is small compared with $g$ then the phase boundary (5) will only be realized when $\Delta \ll 0$ and the temperature is low. In the normal state under these conditions both the exciton occupation and the effects of the dipole interaction are negligible. The transition temperature
(5) then corresponds to non-interacting bosons with an unusual density of states.

To summarize, we have presented a theory of polariton condensation in the Dicke model. By studying the model at fixed excitation, we have generalized the concept of a polariton condensate from the low-density regime. We have found two states: a normal state of excitons and a condensate of polaritons. The polariton condensate is a superposition of a BCS-like state of the excitons and a coherent state of the photon field, and is favored over the normal state by the dipole coupling. We recover conventional polaritons both as the low-density limit of the condensate and as the linear-response excitations of the normal state.

This work was supported by funding from the Engineering and Physical Sciences Research Council, UK.

[1] C. Weisbuch, M. Nishioka, A. Ishikawa, and Y. Arakawa, Phys. Rev. Lett. 69, 3314 (1992).

[2] P. Senellart and J.Bloch, Phys. Rev. Lett. 82, 1233 (1999).

[3] L. S. Dang et al., Phys. Rev. Lett. 81, 3920 (1998).

[4] S. Pau et al., Phys. Rev. A 54, R1789 (1996).

[5] H. Cao et al., Phys. Rev. A 55, 4632 (1997).

[6] M. Kira et al., Phys. Rev. Lett. 79, 5170 (1997).

[7] F. Tassone and Y. Yamamoto, Phys. Rev. B 59, 10830 (1999).

[8] F. Yura and E. Hanamura, Phys. Rev. B 50, 15457 (1994).

[9] J. J. Hopfield, Phys. Rev. 112, 1555 (1958).

[10] F. Jahnke et al., Phys. Rev. Lett. 77, 5257 (1996).

[11] R. H. Dicke, Phys. Rev. 93, 99 (1954).

[12] K. Hepp and E. H. Lieb, Ann. Phys. (N.Y.) 76, 360 (1973).

[13] V. B. Kir'yanov and V. S. Yarunin, Teoreticheskaya i Matematicheskaya Fizika 51, 456 (1982), [Theoretical and Mathematical Physics 51, 615 (1982)].

[14] C. Comte and P. Nozières, J. Phys. (Paris) 43, 1069 (1982).

[15] V. M. Galitskii, S. P. Goreslavskii, and V. F. Elesin, Zh. Eksp. Teor. Fiz. 57, 207 (1969), [Sov. Phys. JETP 30, 117 (1970)].

[16] V. N. Popov, Functional Integrals and Collective Excitations (Cambridge University Press, Cambridge, England, 1987).

[17] A. O. Caldeira and A. J. Leggett, Phys. Rev. Lett. 46, 211 (1981).

[18] P. B. Littlewood and P. R. Eastham, in Proceedings of the NATO Advanced Research Workshop "Optical Properties of Semiconductor Nanostructures", NATO Sciences High Technology Series (Kluwer, Dordrecht, The Netherlands, in press).

[19] K. Maki, in Superconductivity, edited by R. D. Parks (Marcel Dekker, New York, 1969), Vol. 2, Chap. 18, p. 
1035.

[20] D. G. Lidzey et al., Phys. Rev. Lett. 82, 3316 (1999).

[21] D. G. Lidzey et al., Nature 395, 53 (1998).

[22] D. Gammon et al., Phys. Rev. Lett. 76, 3005 (1996).

[23] H. F. Hess et al., Science 264, 1740 (1994).

[24] N. H. Bonadeo et al., Phys. Rev. Lett. 81, 2759 (1998). 